\title{
Influence of various gases on single bubble sonoluminescence
}

\author{
VIJAY H ARAKERI \\ Department of Mechanical Engineering, Indian Institute of Science, Bangalore 560012, India \\ MS received 24 May 1993
}

\begin{abstract}
Influence of various gases on the intensity of single bubble sonoluminescence has been studied. The gases used were air, oxygen, nitrogen, argon and helium. Among these oxygen gave the brightest intensity with nitrogen giving the least.
\end{abstract}

Keywords. Sonoluminescence; bubble dynamics.

PACS Nos $78 \cdot 60 ; 43 \cdot 25 ; 42 \cdot 50$

Single bubble sonoluminescence (SBSL) $[1,2]$ is the phenomenon of light emission from a single bubble driven into extreme nonlinear motion by an acoustic field. The light emitting bubble could be a source of picosecond flashes [2] if the SBSL intensity can be enhanced sufficiently. Towards this end we have investigated the influence of various gases on the intensity of SBSL. The apparatus used presently has been briefly described by Arakeri [3]. It basically consists of a $500 \mathrm{cc}$ boiling flask driven into one of its resonant modes by a piezoelectric drive. A photomultiplier tube (RCA 4526 ) with a $\mathrm{S}-20$ spectral response and a rise time of about 2 ns is used to measure SBSL intensity. The average of single pulse outputs of the PMT was recorded on a fast digital storage oscilloscope (Tekhind 2230) operated in repetitive sampling mode. The typical record obtained on the oscilloscope was presented earlier [3]. The area under the curve is directly related to the integrated charge output of the PMT and as described by Barber [4], this information can be used to obtain the number of photons emitted per flash. The present system was calibrated to give SBSL intensity in terms of the total number of photons emitted per flash. The maximum values attainable for each experimental condition are presented here.

With the exception of one series of tests, the others used distilled water as the liquid medium. The steps in the preparation of samples with various dissolved gases were to repeatedly degas the liquid to a low gas content value and expose it to the particular gas under study. The gas content was monitored with a Van Slyke apparatus. This repeated degassing and exposure was done to ensure that most (if not all) of the air which was originally present was driven out.

It was previously reported [3] that in air/water system, the SBSL intensity is strongly dependent on the extent of dissolved air content. Further studies indicated that the behaviour is more complex than suggested by the single set of experiments. Some additional results are presented in Figure 1. The behaviour in argon/water system is similar to that in air/water system at lower gas content $(\alpha)$ values. However, at higher $\alpha$, the SBSL intensity in argon/water system seems to reach a constant value. Thus, near saturation conditions the light intensity with argon is higher than that with air. This has been the finding with sonoluminescence (SL) from multi-bubble 


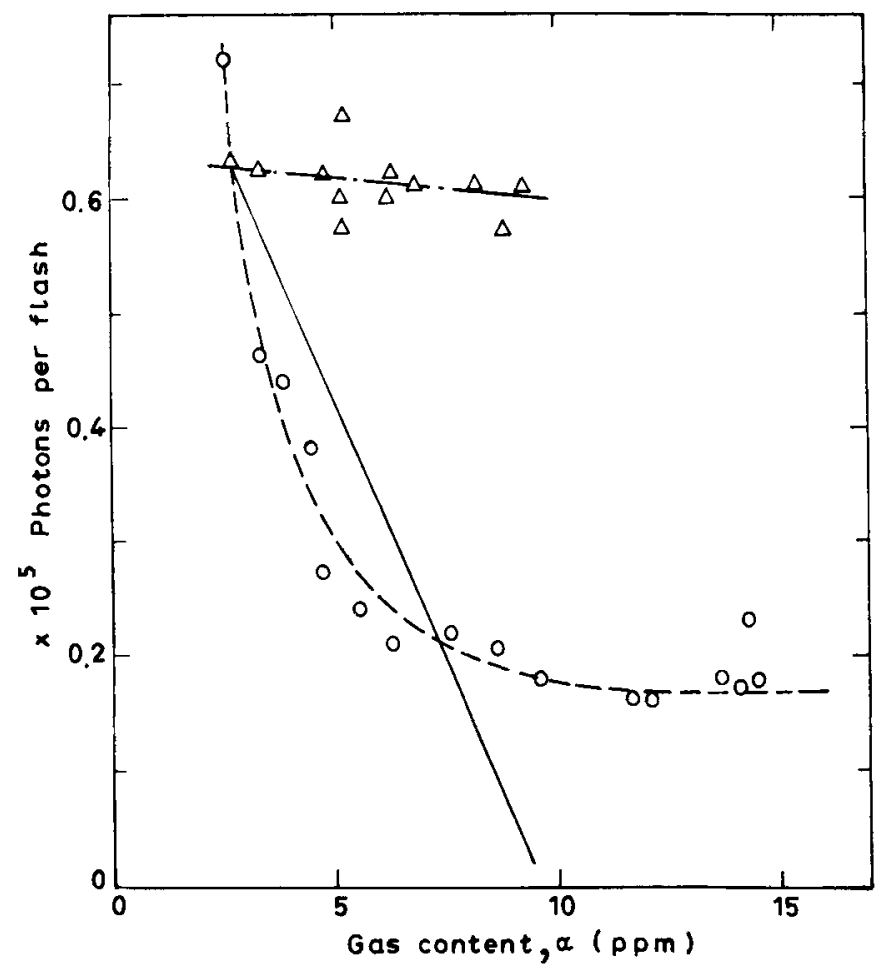

Figure 1. Effect of dissolved air concentration on single bubble sonoluminescence intensity. - air/water (Arakeri [3]), $O$ argon/water, $\Delta$ air $/ 20 \%$ glycerinewater. $T=20 \pm 1^{\circ} \mathrm{C}$.

cavitation fields $[5,6]$. In contrast to air and argon/water systems a surprising finding was that with air/20 per cent glycerine-in-water-solution system, the SBSL intensity hardly changed with $\alpha$ (Figure 1). The presently measured SBSL intensity with this system is near $0.6 \times 10^{5}$ photons per flash. In comparison, Barber and Putterman [2] have measured an intensity of about $0.8 \times 10^{5}$ photons per flash in 25 per cent glycerine-in-water-solution at a frequency of about $30 \mathrm{kHz}$ which is close to our operating frequency. Considering the differences in the apparatus including variations in PMT response, the agreement between the two measurements can be considered to be good. The presently observed difference in the behaviour of air/water and air/glycerine solution systems is quite puzzling. One observation could be relevant here; in the case of air/water system the SBSL was found to be absolutely steady; whereas, with air/glycerine solution system the SBSL would blink i.e. it would go on and off every few seconds. This fact has also been reported by Barber and Putterman [2]. In view of this, water was selected as the liquid medium for further tests.

It has already been noted that SBSL intensity depends on $\alpha$; in addition, Hiller et al [7] have shown that SBSL intensity is also dependent on temperature, T. Comparison of results shows that the latter dependence is stronger than the former, in the sense that SBSL intensity can vary much more for $1^{\circ} \mathrm{C}$ change in $T$ than for $1 \mathrm{ppm}$ change in $\alpha$. For this reason, the influence of various gases on SBSL intensity was studied by varying the sample temperature with a fixed initial value of dissolved gas content of about 5-6 ppm for all the tests. The results are presented in figure 2 . It is clear that with oxygen, the SBSL intensity is the maximum and with nitrogen the minimum. 


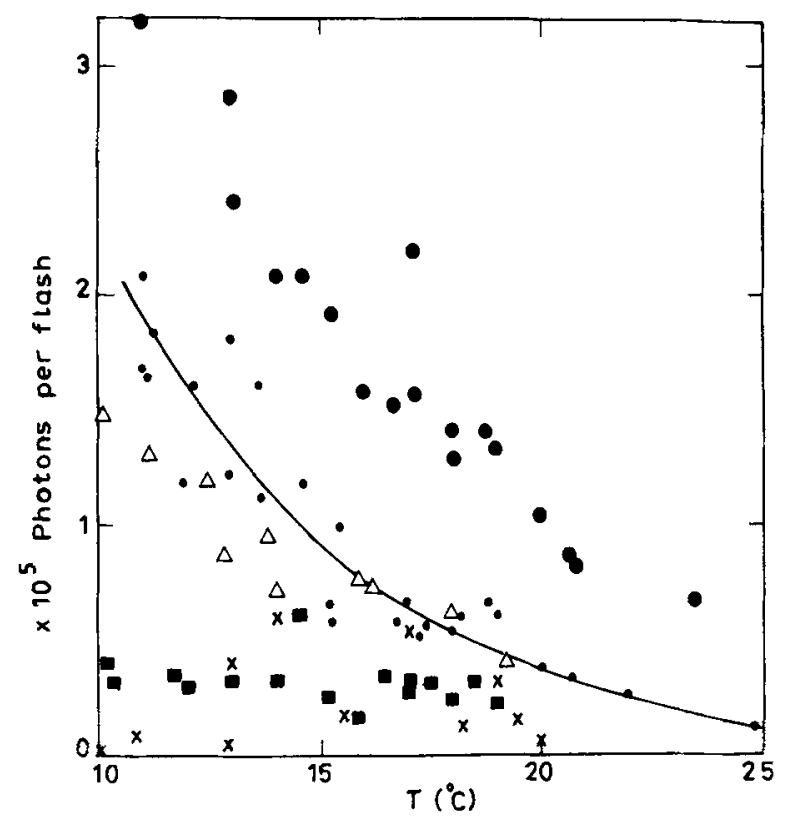

Figure 2. Influence of various gases on maximum single bubble sonoluminescence intensity in water at different temperatures. $\bullet$ air, $\bigcirc$ oxygen, $\Delta$ argon, $\mathbf{D}$ helium, $x$ nitrogen. Solid line is mean through air data. $x=5-6 \mathrm{ppm}$. The intensity levels with air above are significantly lower than those reported in [7] since in the present apparatus most of the ultraviolet rays are cut-off.

The monoatomic gases argon and helium with higher $\gamma$ values show lower intensities than air or oxygen. Close observation of bubble behaviour under light emitting conditions showed that the motion was steady and fixed with air, $\mathrm{O}_{2}$ and $\mathrm{N}_{2}$; whereas, it was unsteady with $\mathrm{Ar}$ and also with $\mathrm{He}$ bubbles. This may partly explain the observed relatively low values of SBSL intensity in the case of He. The intensity difference between the $\mathrm{O}_{2}$ and $\mathrm{N}_{2}$ bubbles is quite significant and is genuinely associated with internal dynamics since the bubble behaviour appeared very similar. In fact additional tests were carried out with $\mathrm{O}_{2}$ and $\mathrm{N}_{2}$ bubbles at higher dissolved gas concentration. In the case of $\mathrm{O}_{2}$ with $\alpha=14 \mathrm{ppm}$, the intensity levels were similar to those for $\alpha=5-6 \mathrm{ppm}$ and are presented in figure 2 . On the other hand with $\mathrm{N}_{2}$ and $\alpha=9.5 \mathrm{ppm}$, no emission could be detected within a temperature range of 10 to $20^{\circ} \mathrm{C}$ of the liquid. It may be possible that the emission band was outside the S-20 spectral range. Present observations do suggest that closer examination of the $\mathrm{O}_{2}$ and $\mathrm{N}_{2}$ bubble behaviour with laser light scattering $[1,8]$ technique and SL spectrum measurement [7] could be very useful.

Previously, influence of various gases on SL from multi-bubble cavitation fields has been investigated [5], including detailed SL spectrum measurements by Sehgal et al [6]. However, all of these measurements have been limited to near saturation conditions and hence direct comparison of present finding in figure 2 with these may not be appropriate. In addition it is not clear whether the phenomenon of SBSL and SL from multi-bubble cavitation fields are similar. 
Vijay $H$ Arakeri

\section{Acknowledgement}

The author would like to thank S Chakraborty for assistance in the initial phases of this study.

\section{References}

[1] D F Gaitan, L A Crum, C C Chruch and R A Roy, J. Acoust. Soc. Am. 91, 3166 (1992)

[2] B P Barber and S J Putterman, Nature (London), 352, 318 (1991)

[3] V H Arakeri, Pramana - J. Phys. 40, L145 (1993)

[4] B P Barber, Synchronous picosecond sonoluminescence (Ph.D. Thesis, Univ. of California at Los Angeles (1992))

[5] A J Walton and G T Reynolds, Adv. Phys. 33, 595 (1984)

[6] C Sehgal, R G Sutherland and R E Verrall, J. Phys. Chem. 84, 388 (1980)

[7] R Hiller, S J Putterman and B P Barber, Phys. Rev. Lett. 69, 1182 (1992)

[8] B P Barber and S J Putterman, Phys. Rev. Lett. 69, 3839 (1992) 